Office of Scientific Research, U.S.A., which through contract number AF 49(638)-807 with the University of Michigan and later with a grant to the University of California, Los Angeles, assisted the program after the observations had been obtained.

\title{
References
}

Aller, L. H. (1954a).-_."Astrophysics: Nuclear Transformations, Stellar Interiors and Nebulae." (Ronald Press Co.: New York.)

Aller, L. H. (1954b).-Ap. J. 120 : 401-12.

Aller, L. H. (1956)._-“Gaseous Nebulae." (Wiley: New York.)

Aller, L. H., and Faulkner, D. J. (1962).-P.A.S.P. 74 : 219-22.

Aller, L. H., and Liller, W. (1959).-Ap. J. 130 : 45-56.

Ambartsumian, V. A. (1933).-Zs.f. Ap. $6: 107-13$.

Burgess, A. (1958).-M.N. 118 : 477-95.

Doherty, L., Henize, K. G., and Aller, L. H. (1956).-Ap. J. Suppl. 2: 345-63.

Henize, K. G. (1956).-Ap. J. Suppl. 2: 315-44.

Henize, K. G., and Westerlund, B. E. (1963).-Ap. J. 137: 747.

JoHNSON, H. M. (1959).-P.A.S.P. 71 : 425-34.

Johnson, H. M. (1961).-P.A.S.P. 73: 20-9.

LiNDSAY, E. M. (1961).-A. J. 66: 169-85.

MATHIS, J. S. (1962).-Ap. J. 136: 374-80.

NaIL, V., Whitvey, C. A., and WAde, C. M. (1953).-Proc. Nat. Acad. Sci. (Washington) 39: $1168-76$.

Seaton, M. J. (1960).-Rep. Progr. Phys. 23 : 313-54.

Seaton, M. J., and Osterbrock, D. E. (1957).-Ap. J. 125 : 66-83.

Shapley, H., and Wilson, H. H. (1925a).-Harvard Obs. Circular No. 271.

Shapley, H., and Wilson, H. H. (1925b).-Harvard Obs. Circular Nos. 275, 276.

WILLSTROP, R. V. (1960).-M.N. 121 : 17-40.

\section{A COMPARISON OF THE 30 DORADUS AND $\eta$ CARINAE NEBULAE}

\author{
D. J. FAulkner
}

Mount Stromlo Observatory

\section{Introduction}

The program of observations of the 30 Doradus and $\eta$ Carinae nebulae described here had its origin in the general study of Magellanic Cloud nebulae considered in the previous paper. The 30 Doradus nebula is considerably larger and brighter than the other HII regions in the Clouds, and may be treated in greater detail. The $\eta$ Carinae nebula was chosen as a comparison object in our own Galaxy.

Most of the results obtained apply to the particular nebulae themselves, and so fall outside the scope of this Symposium. There is one aspect, however, that of the relative abundance of elements in the two systems, which seems to have sufficiently broad implications to warrant inclusion.

\section{Observations of the Spectra of the Nebulae}

Detailed photoelectric spectrophotometer scans of the blue spectral range were obtained for a bright region in each of the two nebulae. The scanner used was that of the University of Michigan, constructed by Liller (1957). Plates 1 and 2 show 
$\mathrm{H} \propto$ photographs of the nebulae on which the setting positions are indicated by rectangles. In the case of the 30 Doradus nebula the brightest part of the great loop was examined, while for the $\eta$ Carinae nebula a position $3^{\prime}$ to the north of the star $\eta$ Carinae was chosen. Figure 1 shows a spectrophotometer trace of the 30 Doradus nebula, from the $3727 \AA$ doublet of [OII] to $\mathrm{H} \delta$ at $4102 \AA$. The pass-band used for the scans is also indicated.

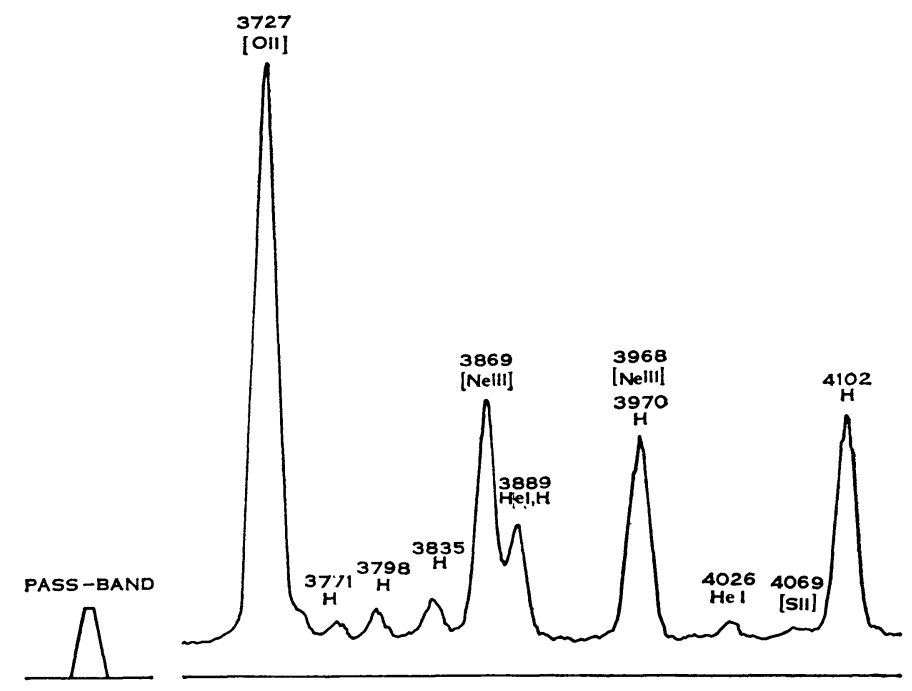

Fig. 1.-A spectrophotometer trace of the 30 Doradus nebula.

The scanner results were supplemented by two or three spectrograms of each object, taken with the 8-in. focal length camera of the Mount Stromlo coudé spectrograph (dispersion $40 \AA / \mathrm{mm}$ ). These were used principally to separate lines too close to be resolved in the scanner observations. A combination of the results of the photoelectric and photographic spectrophotometry gave relative intensities for 24 lines in the case of the 30 Doradus nebula and for 17 lines in the case of the $\eta$ Carinae nebula:.

\section{Correction for Interstellar Reddening}

Before the observed relative intensities of lines may be used for the calculation of abundance, they must be adjusted for the effects of interstellar reddening. This was done by comparing the observed Balmer decrement in each case with the theoretical decrement of Burgess (1958); the $10,000^{\circ} \mathrm{K}$, case $\mathrm{B}$, theoretical values were used. The decrement is so insensitive to temperature that the use of the $10,000^{\circ} \mathrm{K}$ results would cause negligible error, even if the actual electron temperature were considerably different from $10,000^{\circ} \mathrm{K}$.

To achieve the theoretical decrement, a visual absorption of $1 \mathrm{~m} 0$ was required for the 30 Doradus nebula, while $1 \frac{\mathrm{m} .7}{}$ was needed for the $\eta$ Carinae nebula. The relative intensities of the lines, corrected for the reddening corresponding to these absorptions, are shown in Table 1. The last column of the table indicates the theoretical values of Burgess. 
The mean errors in the relative intensities ranged from $4 \%$ for the strongest lines to $10 \%$ for the weakest, while a few lines (marked with colons in Table 1) have even greater uncertainties.

TABLE 1

RELATIVE INTENSITIES

\begin{tabular}{|c|c|c|c|c|}
\hline$\lambda$ & Line & $\begin{array}{c}\text { Intensity } \\
\mathbf{3 0} \\
\text { Doradus }\end{array}$ & $\begin{array}{l}\text { Intensity } \\
\eta \text { Carinae }\end{array}$ & $\begin{array}{c}\text { Intensity } \\
\text { Burgess } \\
(1958)\end{array}$ \\
\hline 3712 & $\mathbf{H}$ & $1 \cdot 8$ & & \\
\hline 3722 & $\mathrm{H}$ & $1 \cdot 9$ & & \\
\hline 3726 & [OII] & 67 & 82 & \\
\hline 3729 & [OII] & 72 & 87 & \\
\hline 3734 & $\mathrm{H}$ & $2 \cdot 1$ & & \\
\hline 3750 & $\mathrm{H}$ & $\mathbf{3} \cdot \mathbf{3}$ & & \\
\hline 3771 & $\mathrm{H}$ & $3 \cdot 7$ & $4 \cdot 8$ & \\
\hline 3798 & $\mathrm{H}$ & $5 \cdot 2$ & $5 \cdot 7$ & $6 \cdot 1$ \\
\hline 3820 & $\mathrm{HeI}$ & 0.9 & & \\
\hline 3835 & $\mathrm{H}$ & $7 \cdot 3$ & $9 \cdot 4$ & $8 \cdot 1$ \\
\hline 3869 & {$[\mathrm{NeIII}]$} & $35 \cdot 2$ & $12 \cdot 5$ & \\
\hline 3889 & $\mathrm{HeI}$ & $6 \cdot 7$ & $7 \cdot 8$ & \\
\hline 3889 & $\mathrm{H}$ & $11 \cdot 4$ & $11 \cdot 4$ & $11 \cdot 4$ \\
\hline 3968 & {$[\mathrm{NeIII}]$} & $10 \cdot 0$ & $2 \cdot 8:$ & \\
\hline 3970 & $\mathrm{H}$ & $18 \cdot 4$ & $17 \cdot 9$ & $17 \cdot 2$ \\
\hline 4026 & $\mathrm{HeI}$ & $2 \cdot 3$ & $4 \cdot 1$ & \\
\hline 4069 & [SII] & $1 \cdot 2:$ & & \\
\hline 4102 & $\mathrm{H}$ & $28 \cdot 3$ & $27 \cdot 0$ & $27 \cdot 6$ \\
\hline 4340 & $\mathbf{H}$ & $48 \cdot 3$ & $48 \cdot 0$ & $48 \cdot 9$ \\
\hline 4363 & [OIII] & $4 \cdot 3$ & & \\
\hline 4471 & $\mathrm{HeI}$ & $4 \cdot 0$ & $5 \cdot 9$ & \\
\hline 4861 & $H$ & 100 & 100 & 100 \\
\hline 4959 & [OIII] & 170 & 66 & \\
\hline 5007 & [OIII] & 477 & 201 & \\
\hline
\end{tabular}

\section{The Electron Temperature and Electron Density}

The electron densities of the regions may be calculated from the relative intensities of the [OII] $3727 \AA$ doublet lines in the usual way; values of $560 \mathrm{~cm}^{-3}$ and $610 \mathrm{~cm}^{-3}$ were found for the 30 Doradus and $\eta$ Carinae nebulae respectively. Similarly the intensity ratio of the $[\mathrm{OIII}]$ lines, $I(4363) / I(4959+5007)$, yields the electron temperature; a value of $10,800^{\circ} \mathrm{K}$ was obtained for the 30 Doradus nebula. Unfortunately, the present observations do not permit an accurate estimate of the $4363 \AA$ line intensity in the $\eta$ Carinae nebula so that no precise value for the electron temperature is available, although it is known to lie in the neighbourhood of $10,000^{\circ} \mathrm{K}$; additional observations will soon be made to provide a better estimate.

We may now consider the abundances of helium and oxygen relative to that of hydrogen. These are the only elements for which we see lines from a sufficient number of ions to estimate the total abundance. 


\section{The Helium Abundance}

Before we may use the intensities of the HeI triplet lines of Table 1 to obtain the abundance of $\mathrm{He}^{+}$, we must examine a suggestion by Pottasch (1962) that diffuse nebulae may become optically thick to the helium triplet lines which have the metastable $2 \mathrm{~s}^{3} \mathrm{~S}_{1}$ level as the lower level. When this happens the relative intensity of the $3889 \AA$ line, for example, is considerably reduced. The intensities of the lines of the $n^{3} \mathrm{D}-2{ }^{3} \mathrm{P}^{\circ}$ series, on the other hand, are relatively unchanged. Thus by comparing the intensity of the $3889 \AA$ line with those of 4471,4026 , and $3820 \AA$, we may determine the optical depth of the nebula in the $3889 \AA$ line. For the 30 Doradus nebula we find

while for the $\eta$ Carinae nebula

$$
\tau(3889)=4,
$$

$$
\tau(3889)=6 .
$$

Tables 1 and 2 of Pottasch's paper then enable us to correct the intensities of the $n^{3} \mathrm{D}-2^{3} \mathrm{P}^{\circ}$ lines for the effects of finite optical depth. The corrections are small, amounting to only $2 \%$ in the worst case.

Abundance estimates may be obtained from the relative intensities of recombination lines using the usual formula, which involves the transition probabilities, the statistical weights of the levels, the excitation and ionization potentials, the electron temperature, and the deviations of the level populations from the thermodynamic equilibrium values, $b_{j}$. Since the lines of the $n^{3} \mathrm{D}-2^{3} \mathrm{P}^{\circ}$ series of helium are very nearly hydrogenic, we may use the $b_{j}$ values of Burgess (1958) for the helium lines, as well as for $\mathrm{H} \beta$.

When the numerical values for the atomic parameters are inserted, the equations for obtaining abundance reduce to

$$
\begin{aligned}
\frac{T_{\mathrm{e}}=10,000^{\circ} \mathrm{K}}{N\left(\mathrm{He}^{+}\right)} & =1.86 \frac{I(4471)}{I(\mathrm{H} \beta)}=2.06 \frac{T_{\mathrm{e}}=20,000^{\circ} \mathrm{K}}{I(\mathrm{H} \beta)} \\
& =3.5 \frac{I(4026)}{I(\mathrm{H} \beta)}=3.8 \frac{I(4026)}{I(\mathrm{H} \beta)} \\
& =6.0 \frac{I(3820)}{I(\mathrm{H} \beta)}=6.4 \frac{I(3820)}{I(\mathrm{H} \beta)}
\end{aligned}
$$

It will be noticed that the expressions for helium abundance are only weakly dependent upon the electron temperature.

Substituting the observed intensities in the $10,000^{\circ} \mathrm{K}$ equations, and taking a weighted mean in each case, we obtain

for the 30 Doradus nebula, and

$$
\frac{N\left(\mathrm{He}^{+}\right)}{N\left(\mathrm{H}^{+}\right)}=0.074 \pm 0.006
$$

$$
\frac{N\left(\mathrm{He}^{+}\right)}{N\left(\mathrm{H}^{+}\right)}=0 \cdot 114 \pm 0 \cdot 018
$$

for the $\eta$ Carinae nebula. The errors shown are based on the mean errors obtained for the several observations of the individual line intensities. 
When discussing the helium abundance, it is frequently assumed (e.g. Mathis (1962)) that the singly charged ion is the only significantly populated ion of the element, so that the abundance $N\left(\mathrm{He}^{+}\right) / N\left(\mathrm{H}^{+}\right)$is in fact the total abundance, $N(\mathrm{He}) / N(\mathrm{H})$. It is known from the absence of the $4686 \AA$ line in diffuse nebulae that they contain a negligible amount of doubly ionized helium. Furthermore, if observations are made of the central part of a nebula near the exciting stars, the amount of neutral helium will also be low. Thus this approximation, although not valid for the low excitation parts of diffuse nebulae, may be used when dealing with the brightest parts. The abundance estimates quoted above may thus be regarded as estimates of the total abundance of helium relative to hydrogen.

\section{The Oxygen Abundance}

The ionic abundances of $\mathrm{O}^{+}$and $\mathrm{O}^{2+}$ are easily obtained from the relative intensities of their forbidden lines using the usual formulae. The factors involved are the appropriate transition probabilities and collision strengths, the electron temperature, and the electron density. We have used the numerical values of

TABLE 2

COMPARISON OF ABUNDANCE RESULTS

\begin{tabular}{l|l|l}
\hline \multicolumn{1}{c|}{ Nebula } & $\frac{N(\mathrm{He})}{N(\mathrm{H})}$ & $\frac{N(\mathrm{O})}{N(\mathrm{H})}$ \\
\hline Orion (Galaxy) & $\begin{array}{l}0 \cdot 117 \\
0 \cdot 11\end{array}$ & $3 \cdot 4 \times 10^{-4}$ \\
M8 (Galaxy) & $0 \cdot 094$ \\
M20 (Galaxy) & $0 \cdot 114$ & \\
$\eta$ Carinae (Galaxy) & $0 \cdot 102$ & \\
NGC 604 (M33) & $0 \cdot 073-0 \cdot 08$ & \\
NGC 346 (SMC) & $0 \cdot 074$ & $2 \cdot 5 \times 10^{-4}$ \\
30 Doradus (LMC) & & \\
\hline
\end{tabular}

Seaton (1960). Unlike the helium calculations, those for the oxygen abundance depend strongly on the electron temperature; thus in the case of the $\eta$ Carinae nebula, no estimate for the oxygen abundance may be obtained from the present data. For the 30 Doradus nebula we obtain

$$
\begin{aligned}
& \frac{N\left(\mathrm{O}^{+}\right)}{N\left(\mathrm{H}^{+}\right)}=4 \cdot 1 \pm 0 \cdot 8 \times 10^{-5} \\
& \frac{N\left(\mathrm{O}^{2+}\right)}{N\left(\mathrm{H}^{+}\right)}=1 \cdot 9 \pm 0 \cdot 3 \times 10^{-4} .
\end{aligned}
$$

These two ions comprise most of the oxygen in diffuse nebulae. In the Orion nebula, for example, Aller and Liller (1959) found that only $10 \%$ of the oxygen is contained in other ions. If we adopt the same figure for the 30 Doradus nebula we obtain

$$
\frac{N(\mathrm{O})}{N(\mathrm{H})}=2 \cdot 5 \pm 0 \cdot 6 \times 10^{-4}
$$

where the mean error of the observations has been increased by a further $10 \%$ to allow for the uncertainty in the contribution of the other ions. 
THE 30 DORADUS AND $\eta$ CARINAE NEBULAE

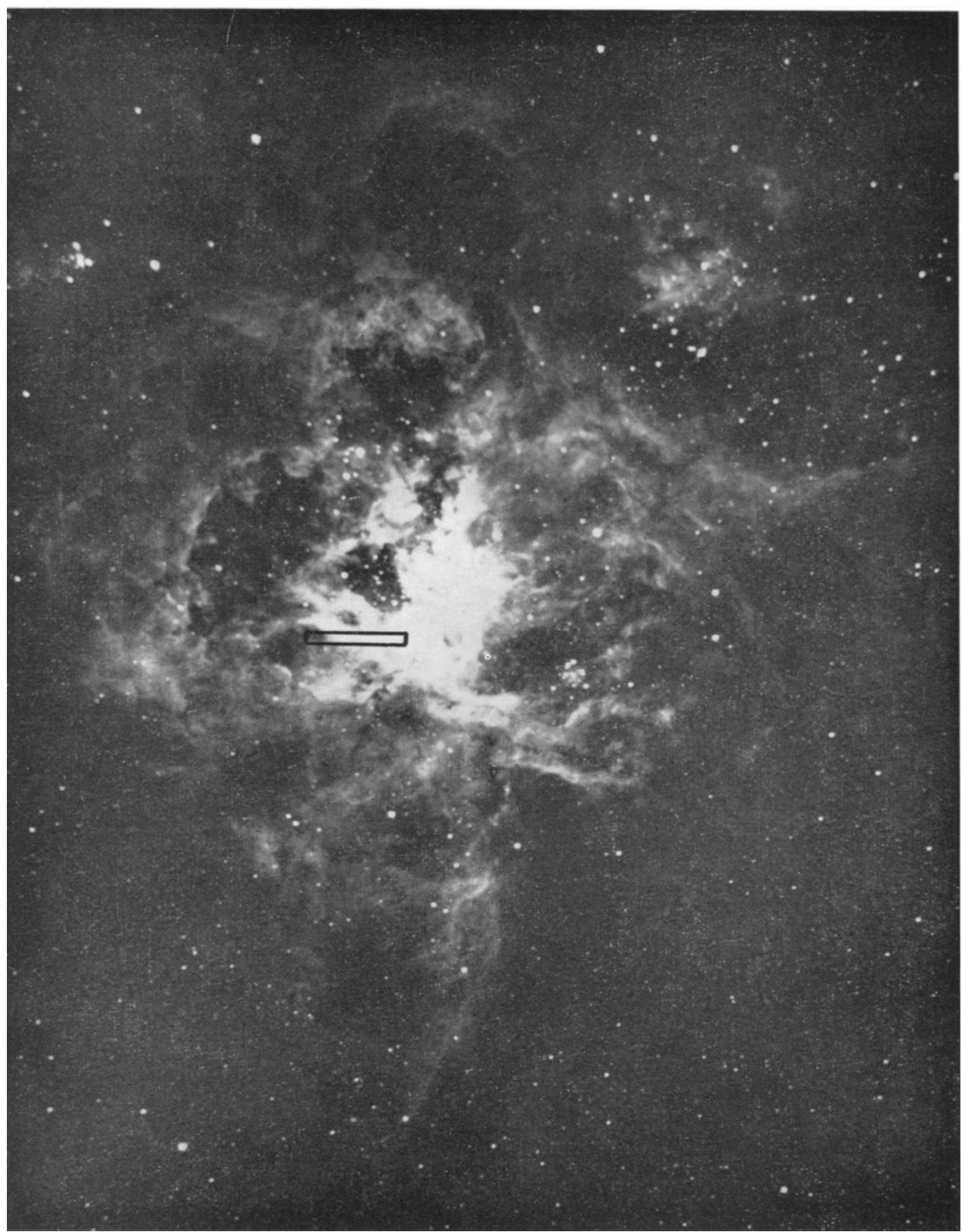

A photograph of the 30 Doradus nebula in $H_{\alpha}$ light. South is to the top and east to the left. The rectangle indicates the size and setting position of the scanner entrance slit used for the detailed spectral scans.

I.A LRSI Symposium No. 20. Paper 64 
THE 30 DORADUS AND $\eta$ CORINAE NEBULAE

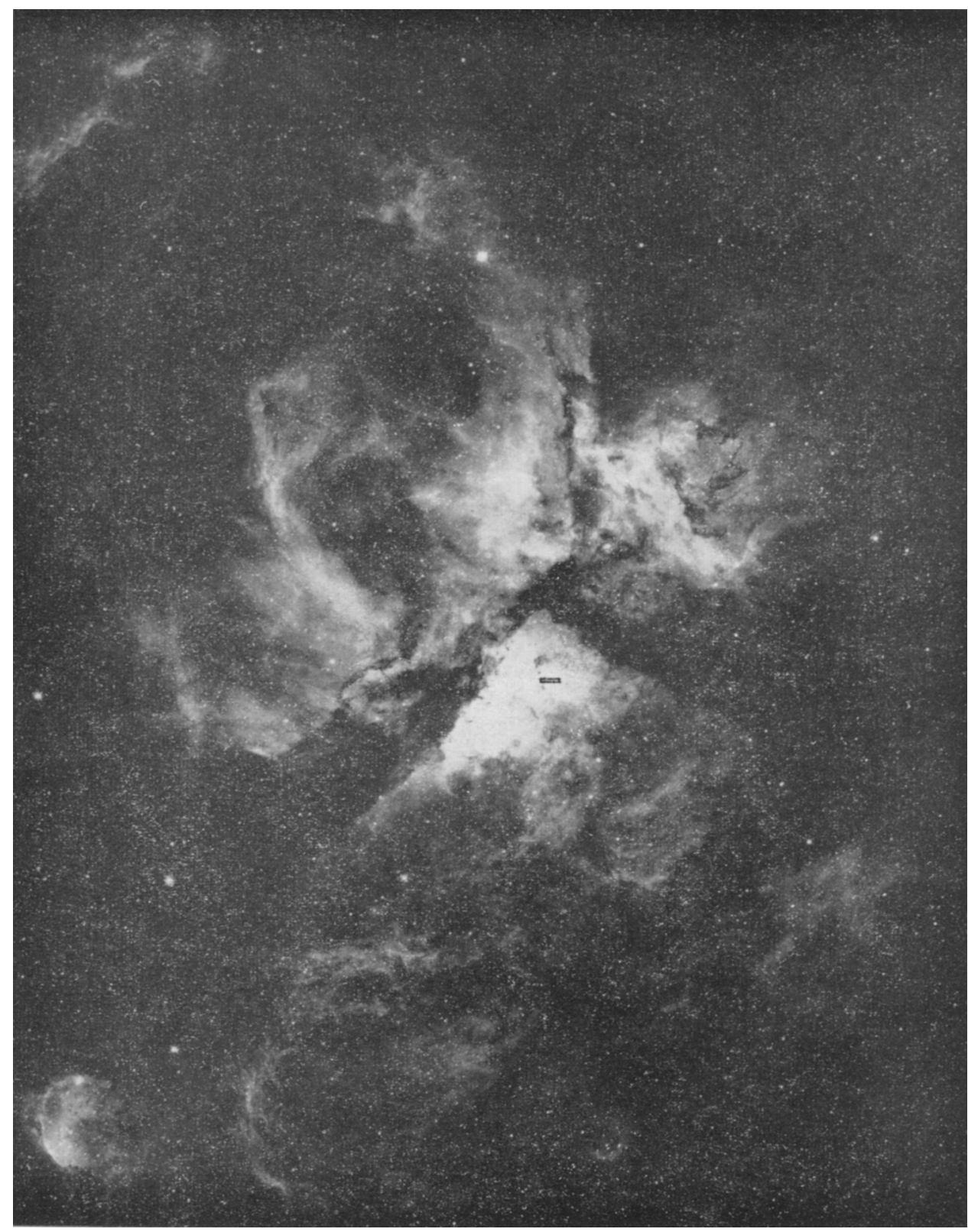

A photograph of the $\eta$ Carinae nebula in $\mathrm{H} \alpha$ light. (The plate was taken by Mr. D. Sher.) South is to the top and east to the right. The rectangle indicates the size and setting position of the scanner entrance slit used for the detailed spectral scans.

IAU URSI Symposium No. 20. Paper 64 


\section{Comparisons with Other Nebulae}

Table 2 provides a comparison of the abundance results obtained in this study with those published for other nebulae.

Mathis (1962) has recently determined the helium abundance in the Orion nebula, M8, M20, and NGC 604. He used exactly the same sources of information to obtain the numerical constants of equation (1) as we have done, and his results should thus be strictly comparable with those found here. Aller and Faulkner (1962) have made a determination of the helium abundance in NGC 346 in the Small Magellanic Cloud, obtaining a value $0 \cdot 11$. In this case, however, the atomic parameters used were slightly different. If, for the sake of uniformity, we use equation (1) to reduce their observed line intensities, the abundance obtained is 0.073 . Interstellar absorption could make this value as high as 0.08 . It thus appears that the abundance of helium in the 30 Doradus nebula, and also in NGC 346, is rather less than that in nebulae in the Galaxy.

In the case of the 30 Doradus nebula, we may also compare the abundance of oxygen found in this investigation with the value of $3 \cdot 4 \times 10^{-4}$ obtained for the Orion nebula by Aller and Liller (1959). For both oxygen and helium, therefore, the 30 Doradus nebula abundance is $25-35 \%$ less than the corresponding Orion nebula abundance, and it seems unlikely that this difference could be accounted for by observational errors.

The author was supported by a CSIRO Post-Graduate Studentship during the first part of this investigation, and by an Australian National University Research Scholarship thereafter.

\section{References}

Aller, L. H., and Faulkner, D. J. (1962).-P.A.S.P. $74: 219-22$.

Aller, L. H., and Liller, W. (1959).-Ap. J. 130: 45-56.

Burgess, A. (1958).-M.N. $118: 477-95$.

LILLER, W. (1957).-P.A.S.P. 69: 511-17.

Mathis, J. S. (1962).-Ap. J. 136: 374-80.

Pottasch, S. R. (1962).-Ap. J. 135 : 385-93.

Seaton, M. J. (1960).-Rep. Progr. Phys. 23 : 313-54.

\section{Discussion}

Pagel: The correction for interstellar reddening depends on a certain theory of the Balmer decrement which could itself be affected by self-absorption in Balmer lines. How sensitive are the abundances of helium and oxygen to the reddening correction?

Faulkner : An optical depth in $\mathrm{H}_{\alpha}$ of the size suggested by Pottasch would cause changes in the calculated value of interstellar reddening which could be large enough to negate the difference found between the helium abundance values in the 30 Doradus and $\eta$ Carinae nebulae. However, Mathis has discussed this question, and has shown that the hypothesis that nebulae are optically thin to the Balmer lines fits his observations best. Furthermore, Osterbrock has demonstrated from theoretical considerations that self-absorption in the Balmer lines may be kept low in diffuse nebulae by the emission of Lyman- $\alpha$ quanta well away from the line centre, followed by their escape from the nebulae without further reabsorption.

Arp: The intermediate age clusters which are in the outer regions of our own Galaxy like NGC 2158 and NGC 7789 show a metal to hydrogen reduction, like the Sun relative to the 
Hyades, of a factor near $50 \%$. It is very interesting to note that the helium to hydrogen ratio and oxygen to hydrogen ratio found by Faulkner and Aller in the Magellanic Clouds are in the same direction, and by what is a fairly comparable amount, i.e. $30 \%$.

Feast: Can Mr. Faulkner say whether a decrease in the adopted interstellar absorption will increase or decrease the $\mathrm{He} / \mathrm{H}$ ratio for 30 Doradus?

Faulkner: Yes, a decrease in the adopted interstellar absorption would decrease the $\mathrm{He} / \mathrm{H}$ ratio which I calculated.

Pagel: A comment on Dr. Arp's statement. Did he say that the Sun is deficient in metals, and if so, could he amplify this statement?

Arp : I judge the metal deficiency of the Sun relative to the Hyades by the three-colour $U, B, V$ photometry.

Pagel: The curve of growth doesn't demonstrate this.

Aller: The $\mathrm{He} / \mathrm{H}$ ratio can be estimated by comparing $\mathrm{HeI} \lambda 4471$ to $\mathrm{H} \gamma \lambda 4340$; the effects of interstellar absorption cannot be important in this case.

Gascoigne: How far are the abundance results dependent on assumptions on the uniformity of the regions studied?

Faulkner: This will vary from nebula to nebula. In the case of the 30 Doradus nebula, the region studied is a loop which appears very uniform, and is considerably brighter than surrounding regions. Thus virtually all the light received is coming from this uniform region, and the effect will be small.

Gascoigne: If the structure were patchy as the Orion nebula is believed to be, in which direction would the abundance be affected?

Aller: This depends on the element considered.

Faulkner : The emission per unit volume in a HeI recombination line is proportional to the product of the number densities of ionized hydrogen and singly ionized helium, $N\left(\mathrm{H}^{+}\right) \cdot N\left(\mathrm{He}^{+}\right)$. Similarly the emission per unit volume in a Balmer line is proportional to $\left[N\left(\mathrm{H}^{+}\right)\right]^{2}$. Thus, provided the ratio $N\left(\mathrm{He}^{+}\right) / N\left(\mathrm{H}^{+}\right)$remains constant throughout the density fluctuations, the relative intensities of the helium and hydrogen recombination lines will be unchanged, and the density fluctuations will have no effect on the calculated abundance of helium.

For oxygen the situation is far more complicated, since here we are dealing with forbidden lines, and the density also plays a part in determining the balance between collisional and radiative de-excitation of the metastable levels.

\title{
65. PLANETARY NEBULAE AND WOLF-RAYET STARS IN THE MAGELLANIC CLOUDS
}

\author{
B. E. WesterLund \\ Mount Stromlo Observatory
}

\section{The Planetary Nebulae}

Objects in the Small Magellanic Cloud classified by Lindsay (1961) as planetary nebulae or probable planetary nebulae have been studied in detail on large-scale photographs (Henize and Westerlund 1963). Of Lindsay's 50 objects, 11 are clearly resolved, 2 are probably resolved, 12 show stellar images, 13 are below the limit of the plates, 11 (fairly faint) are outside the photographed regions, and 1 is of uncertain identification. The masses of the resolved nebulae lie between 2 and 33 solar masses. They are therefore classified as small diffuse nebulae; it appears unlikely that the mass of a planetary nebula can exceed a few tenths of a solar mass. 